\title{
First observations and performance of the RPW instrument on board the Solar Orbiter mission
}

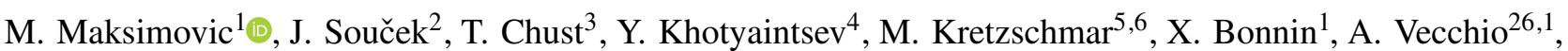 \\ O. Alexandrova ${ }^{1}$, S. D. Bale ${ }^{7,8,9}$, D. Bérard ${ }^{1}$, J.-Y. Brochot ${ }^{5}$, N. J. T. Edberg ${ }^{4}$, A. Eriksson ${ }^{4}$, L. Z. Z Hadid $^{3}$, \\ E. P. G. Johansson ${ }^{4}$, T. Karlsson ${ }^{10}$, B. Katra ${ }^{3}$, V. Krasnoselskikh ${ }^{5}$, V. Krupař ${ }^{11,12}$, S. Lion ${ }^{1}$, E. Lorfèvre ${ }^{13}$,
} L. Matteini ${ }^{14,1}$, Q. N. Nguyen ${ }^{1}$, D. Píša ${ }^{2}$, R. Piberne ${ }^{3}$, D. Plettemeier ${ }^{15}$, H. O. Rucker ${ }^{35}$, O. Santolík ${ }^{2,17}$, K. Steinvall ${ }^{4}$, M. Steller ${ }^{16}, \breve{S}$. Štverák ${ }^{18,2}$, P. Trávníček ${ }^{7,2}$, A. Vaivads ${ }^{10,4}$, A. Zaslavsky ${ }^{1}$, S. Chaintreuil ${ }^{1}$, M. Dekkali ${ }^{1}$, P.-A. Astier ${ }^{1}$, G. Barbary ${ }^{1}$, K. Boughedada ${ }^{1}$, B. Cecconi ${ }^{1}$, F. Chapron ${ }^{1}$, C. Collin ${ }^{1}$, D. Dias ${ }^{1}$, L. Guéguen ${ }^{1}$, L. Lamy ${ }^{19,1}$, V. Leray ${ }^{20}$, L. R. Malac-Allain ${ }^{1}$, F. Pantellini ${ }^{1}$, J. Parisot ${ }^{1}$, P. Plasson ${ }^{1}$, S. Thijs ${ }^{1}$, I. Fratter ${ }^{13}$, E. Bellouard ${ }^{13}$, P. Danto ${ }^{13}$, S. Julien ${ }^{13}$, E. Guilhem ${ }^{21}$, C. Fiachetti ${ }^{13}$, J. Sanisidro ${ }^{13}$, C. Laffaye ${ }^{13}$, F. Gonzalez ${ }^{13}$, B. Pontet ${ }^{13}$, N. Quéruel ${ }^{13}$, G. Jannet ${ }^{5}$, P. Fergeau ${ }^{5}$, T. Dudok de Wit ${ }^{5,6}$, T. Vincent ${ }^{5}$, C. Agrapart ${ }^{5}$, J. Pragout ${ }^{5}$, M. Bergerard-Timofeeva ${ }^{5}$, G. T. Delory ${ }^{9}$, P. Turin ${ }^{9}$, A. Jeandet ${ }^{3}$, P. Leroy ${ }^{22}$, J.-C. Pellion ${ }^{3}$, V. Bouzid ${ }^{3}$, W. Recart ${ }^{3,13}$, I. Kolmašová ${ }^{2,17}$, O. Krupařová ${ }^{11,12}$, L. Uhlî́r ${ }^{2}$, R. Lán ${ }^{2}$, J. Baše ${ }^{2}$, M. André ${ }^{4}$, L. Bylander ${ }^{10}$, V. Cripps ${ }^{4}$, C. Cully ${ }^{23}$, S.-E. Jansson ${ }^{4}$, W. Puccio ${ }^{4}$, J. Bř́nek ${ }^{18}$, H. Ottacher ${ }^{16}$, V. Angelini ${ }^{14}$, M. Berthomier ${ }^{3}$, V. Evans ${ }^{14}$, K. Goetz ${ }^{24}$, P. Hellinger ${ }^{18}$, T. S. Horbury ${ }^{14}$, K. Issautier ${ }^{1}$, E. Kontar ${ }^{25}$, O. Le Contel $^{3}$, P. Louarn ${ }^{27}$, M. Martinović ${ }^{28,29,1}$, D. Müller ${ }^{30}$, H. O’Brien ${ }^{14}$, C. J. Owen ${ }^{31}$, A. Retino ${ }^{3}$, J. Rodríguez-Pacheco ${ }^{32}$, F. Sahraoui ${ }^{3}$, L. Sanchez ${ }^{33}$, A. P. Walsh ${ }^{33}$, R. F. Wimmer-Schweingruber ${ }^{34}$, and I. Zouganelis ${ }^{33}$

(Affiliations can be found after the references)

Received 7 May 2021 / Accepted 20 September 2021

\begin{abstract}
The Radio and Plasma Waves (RPW) instrument on the ESA Solar Orbiter mission is designed to measure in situ magnetic and electric fields and waves from the continuum up to several hundred $\mathrm{kHz}$. The RPW also observes solar and heliospheric radio emissions up to $16 \mathrm{MHz}$. It was switched on and its antennae were successfully deployed two days after the launch of Solar Orbiter on February 10, 2020. Since then, the instrument has acquired enough data to make it possible to assess its performance and the electromagnetic disturbances it experiences. In this article, we assess its scientific performance and present the first RPW observations. In particular, we focus on a statistical analysis of the first observations of interplanetary dust by the instrument's Thermal Noise Receiver. We also review the electro-magnetic disturbances that RPW suffers, especially those which potential users of the instrument data should be aware of before starting their research work.
\end{abstract}

Key words. solar wind - Sun: radio radiation - Sun: general

\section{Introduction}

The Radio and Plasma Waves (RPW) instrument on the ESA Solar Orbiter mission (Müller et al. 2020; Zouganelis et al. 2020) is designed to measure the magnetic and electric fields, the plasma wave spectra and polarization properties, as well as the spacecraft $(\mathrm{S} / \mathrm{C})$ floating potential and solar radio emissions in the interplanetary medium. The full description of the instrument and of its specific science capabilities, which will aid in answering the mission's overarching science objectives, was given by Maksimovic et al. (2020). In particular, RPW has been designed to measure the three-component magnetic field fluctuations from about $3 \mathrm{~Hz}$ to $1 \mathrm{MHz}$ in order to fully characterize magnetized plasma waves in this range. The three RPW electric antennae provide the electric field over a wide frequency range from $\mathrm{DC}$ to $16 \mathrm{MHz}$, covering all relevant wave modes from the MHD range through whistler and Langmuir waves up to solar radio emissions, while also providing the potential of the spacecraft with respect to the surrounding plasma. Finally, the instrument known as the Thermal Noise Receiver (TNR) can detect the local quasi-thermal noise, providing accurate measurements of the in situ absolute electron density and, potentially, of the temperature, when the ambient plasma Debye length is adequate (Meyer-Vernet et al. 2017).

Since the launch of the mission from Cape Canaveral on February 10, 2020, we have acquired enough data to assess the instrument performance and the electromagnetic disturbances it experiences. In Sect. 2, we briefly present the data related to the instrument sensor deployments. In Sect. 3, we present the RPW science performance by describing the numerous first results that have been obtained. In Sect. 4, we focus on the statistical analysis of the first observations of interplanetary dust by the TNR. Finally, in Sect. 5, we describe the electro-magnetic (EM) environment of the instrument and provide a list of the EM disturbances that potential users of RPW data should be aware of.

\section{Sensor deployments}

Right after the launch, the first critical activity for RPW was the deployment of its electric antennae. These took place on February 11, 2020 at 09:33:11 for the PZ/Ant1 antenna (see Fig. 7 in Maksimovic et al. 2020 for the antennae denomination) and on February 13, 2020 at 00:24:15 and 00:48:40 for 


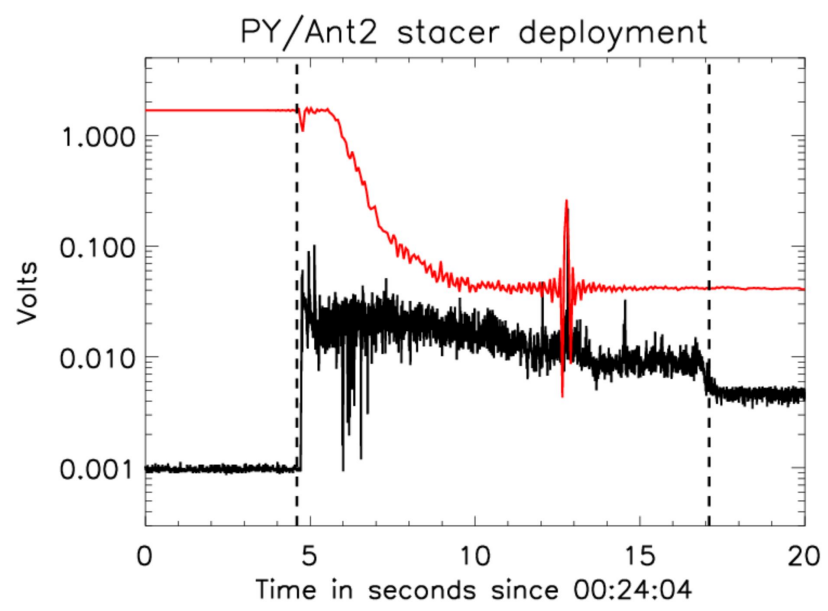

Fig. 1. LFR PY-PZ probe-to-probe electric potential (in red) and TDS time series of the PY maximum amplitude electric potential (in black). Since the PZ antenna was already deployed at the time of the PY antenna deployment, these two combined quantities are good indicators of the duration of the PY stacer deployment, which was $12.5 \mathrm{~s}$ (the time interval between the two dashed vertical lines).

the PY/Ant2 and MY/Ant3 antennae, respectively. As described in more detail in Maksimovic et al. (2020), the three RPW electric antennae consist each of a $1 \mathrm{~m}$ rigid deployable boom and a $6.5 \mathrm{~m}$ stacer deployable monopole, which is actually the electric sensor itself.

The boom and stacer deployments were commanded separately, as planned. For each of the three antennae, once the boom has been deployed and has completed its ninety-degree rotation, the stacer monopole has been deployed by means of a commanded Frangibolt (Maksimovic et al. 2020). The design of a stacer is such that it deploys under its own power until it reaches the end of travel, with its speed and final length controlled by means of a cable and a flyweight brake. All the deployments of RPW stacers on ground, in conditions simulating the absence of gravity, showed that the typical duration of the deployment for a $6.5 \mathrm{~m}$ long stacer was on the order of $12 \mathrm{~s}$. In order to ensure that the full length of the stacers was deployed in flight, we analyzed the RPW data recorded during the respective deployments. Figure 1 shows these data for PY/Ant2 antenna. In this figure, we display the Low Frequency Receiver (LFR) PY-PZ probe to probe electric potential (in red) and the Time Domain Sampler (TDS) time series of the PY maximum amplitude electric potential (in black). Since the PZ antenna was already depoloyed at the time of the PY antenna deployment, these two quantities combined are good indicators of the duration of the PY stacer deployment. As we can see in Fig. 1, the duration of the PY/Ant2 stacer deployment was $12.5 \mathrm{~s}$ (the time interval between the two dashed vertical lines). We also analyzed (not shown here) the data for the MY/Ant3 deployment, which also show the full deployment. As for the PZ/Ant1 stacer, however, due to a Solar Orbiter safe mode transition on February 11, 2020, the RPW telemetry for this deployment has been lost. Finally, note that some of the Antenna radio-electrical properties provided in Fig. 9 of Maksimovic et al. (2020) are not correct. The new figure with the corrected parameters is provided in Fig. 1 of Maksimovic et al. (2021).

The second critical activity for RPW, as well as for the MAG (Horbury et al. 2020) and SWA (Owen et al. 2020) instruments, was the Solar Orbiter instrument boom deployment. Indeed, the RPW Search-Coil Magnetometer (SCM), as well as the two lat- ter instruments, are located on this instrument boom, two meters away from the spacecraft body for the SCM. The reason for this location is that, as for MAG, the SCM must be kept away from the spacecraft in order to minimize the magnetic contamination caused by this latter. The instrument boom deployment took place in two steps, on February 12, 2020 around 19:03 and 19:04 and occurred nominally. The SCM data gathered around this deployment (not displayed here) shows that the S/C magnetic noise measured by the SCM is reduced by at least a factor of 10 , with respect to the folded configuration of the instrument boom. Further data recorded by RPW during low solar wind magnetic activity have shown that the required SCM sensitivity has been reached in space. This will be presented in a forthcoming article.

\section{RPW first results and science performance}

Overall the RPW science performance is very good, as evidenced by the large number of studies conducted with data from the instrument and published in this special issue (Berčič et al. 2021; Carbone et al. 2021; Chust et al. 2021; Dimmock et al. 2021; Graham et al. 2021; Hadid et al. 2021; Khotyaintsev et al. 2021; Kretzschmar et al. 2021; Matteini et al. 2021; Musset et al. 2021; Ṕ́śa et al. 2021; Soucek et al. 2021; Steinvall et al. 2021; Vecchio et al. 2021; Zaslavsky et al. 2021). Below, we summarize part of our results, which are linked to the main scientific objectives of the instrument and reported in these articles.

\subsection{Low-frequency measurements and turbulence}

One of the science objectives of the Solar Orbiter mission is to clarify the physical mechanisms responsible for the acceleration and heating of the solar wind. It is generally believed that the extra energy, in addition to that of the coronal thermal, which is necessary to achieve the heating could have its origin in the turbulent electromagnetic fluctuations of the solar wind (Belcher \& Davis 1971; Tu \& Marsch 1995; Bruno \& Carbone 2013; Alexandrova et al. 2013). As for the basic physical processes responsible for this heating and conversion of thermal energy into directed kinetic energy, it is generally believed that complex mechanisms that couple particles with electromagnetic fluctuations, in the form of low-frequency waves or coherent structures, are at work (Sperveslage et al. 2000; Greco et al. 2009; Tsurutani et al. 2011; Lion et al. 2016).

When designing the RPW instrument, it was important to include the capability to measure high-quality electric fields and density fluctuations up to frequencies of at least about $100 \mathrm{~Hz}$ (Vaivads et al. 2007). Indeed, on RPW, a current bias is applied to each of the antennae in order to bring their potentials closer to the local plasma potential. This enables RPW to make sensitive measurements of the DC (Steinvall et al. 2021) and low-frequency electric fields (Chust et al. 2021; Kretzschmar et al. 2021; Graham et al. 2021) and the spacecraft potential (Khotyaintsev et al. 2021). Steinvall et al. (2021) report that the low-frequency electric field provided by RPW is of such high quality that by using the de Hoffmann-Teller analysis (de Hoffmann \& Teller 1950; Sonnerup et al. 1987), Solar Orbiter's magnetic and electric field measurements can be used to estimate the solar wind speed when plasma data is unavailable.

Moreover, the high quality of the RPW electron density deduced from the spacecraft potential has been used by Carbone et al. (2021) to study and quantify the properties of 
turbulence in the solar wind during the first months of the Solar Orbiter mission. These authors have found that the Kolmogorov scaling of the density fluctuations is only present in part of the analyzed solar wind samples. Other intervals, which are not described by models of turbulence, are observed predominantly during intervals of enhanced ion frequency wave activity, as observed by the MAG instrument (Horbury et al. 2020).

\subsection{Whistler waves in the solar wind}

Chust et al. (2021), Kretzschmar et al. (2021) performed a detailed analysis of whistler waves that have been aptly measured by RPW. The polarisation and phase velocity of these waves could be reliably determined in the plasma frame. Following these studies, the onboard computation of the waves parameters, the so-called basic parameters (Maksimovic et al. 2020), have been validated. These studies have also revealed a constant phase shift of about $50^{\circ}$ between the measurements of the $E$ and $B$ fields at frequency between a few Hertz up to $70 \mathrm{~Hz}$, and possibly also at higher frequencies, where the observation of physical signals has been rare up to now.

\subsection{Quasi-thermal noise and density from the spacecraft potential}

The accurate in situ measurement of the properties of the solar wind electrons is a key element for understanding the physics of the solar wind. For this purpose, the quasi-thermal noise (QTN) spectroscopy technique is a very robust method since it is based on the use of a passive electric antenna for measuring the electrostatic field spectrum produced by the electron and ion thermal motions in a stable plasma (Meyer-Vernet et al. 2017). The QTN spectroscopy requires an antenna length, $L$, larger than the local Debye length, $L_{\mathrm{D}}$, in order to better detect the plasma peak and to adequately measure the electron kinetic temperature.

The first RPW observations of the QTN plasma peak are quite encouraging, showing the presence of the plasma peak about $70 \%$ of the observation time. When the TNR does not suffer from electro-magnetic contamination and when the spacecraft is in a dense enough environment so that $L \geq L_{\mathrm{D}}$, an adequate dynamic spectrum as the one on the upper panel of Fig. 2 can be observed. In this dynamic spectrum, the plasma frequency can clearly be seen and varies between roughly 55 and $75 \mathrm{kHz}$. Using a peak tracking technique, based on the detection of the strongest gradient of the power spectral density $\partial\left(V^{2} / \mathrm{Hz}\right) / \partial f$, it is possible to detect the plasma frequency, $f_{\mathrm{p}}$ (in $\mathrm{kHz}$ ), and deduce the temporal variation of the local electron density, (in $\left.\mathrm{cm}^{-3}\right) N_{\mathrm{e}-f_{\mathrm{p}}}=\left(f_{\mathrm{p}} / 9\right)^{2}$. Here, $N_{\mathrm{e}-f_{\mathrm{p}}}$ is displayed on the lower panel of Fig. 2 in red. As can be seen from the figure, the QTN electron density is discretized because of the frequency resolution of the TNR. Typically, we have thus an uncertainty on the order of $\delta N_{\mathrm{e}-f_{\mathrm{p}}} / N_{\mathrm{e}-f_{\mathrm{p}}}=2 \delta f_{\mathrm{TNR}} / f_{\mathrm{TNR}} \simeq 0.086$. Superimposed in black on the lower panel of Fig. 2, we have the electron density, $N_{\mathrm{e}-\mathrm{S} / \mathrm{C}}$, deduced from the spacecraft potential (Khotyaintsev et al. 2021), after calibration to $N_{\mathrm{e}-f_{\mathrm{p}}}$ over a much longer time interval. In this way, by combining the absolute accuracy of the TNR technique with the high resolution, fast sampling, and insensitivity to high frequency noise of the spacecraft potential measurement, RPW can provide a plasma density data product of very high quality and scientific value.

The next step of the QTN implementation on RPW will be to implement a comprehensive fitting of the full spectrum between 4 and about $100 \mathrm{kHz}$ in order to avoid the contamination from the spacecraft PCDU. This full fitting should provide a better accuracy on the deduced density, with uncertainties closer to those obtained in previous missions (Maksimovic et al. 1995, 1998), in addition to the measurement of the core electron temperature (Issautier et al. 1999).

\subsection{Solar Radio bursts and associated Langmuir waves}

Despite the EM contamination of the RPW radio frequency domain, as discussed in Sect. 5, it is still possible to observe solar Type III bursts, in particular in the lower frequency part of the spectrum below $1 \mathrm{MHz}$, where several clean frequency bands remain (Vecchio et al. 2021). The Solar Orbiter radio observations add another measurement point for triangulation of the Type III source regions, in addition to WIND, STEREO, and Parker Solar Probe (Musset et al. 2021). The combination of observations from those four points distributed in the heliosphere allows for a precise tracking and characterization of the azimuthal structure of the Type III events (Gómez-Herrero et al. 2021).

On multiple occasions in 2020, Solar Orbiter encountered so-called in situ Type III events, where the source region swept past the spacecraft. During these crossings, the EPD instrument (Rodríguez-Pacheco et al. 2020) registered energetic solar electrons and, at the same time, RPW observed electrostatic Langmuir waves at the local electron plasma frequency. These waves are responsible for the transfer of energy from the electron beam to the radio emission (Gómez-Herrero et al. 2021). In Soucek et al. (2021) another example of a well-resolved series of in situ Type III bursts observed by RPW is presented. In this example, it was demonstrated that the TDS subsystem successfully identified the local plasma waves in its statistical data and captured waveform snapshots with different polarization properties (both linear and transverse).

\subsection{Interplanetary shocks and discontinuities}

As described by Maksimovic et al. (2020), a shock detection algorithm was implemented on board RPW. This algorithm is based on the real-time joint analysis of the magnetic field vector provided by the MAG instrument (Horbury et al. 2020) and the proton number density and bulk velocity provided by the SWA instrument (Owen et al. 2020). This technique, described in detail by Kruparova et al. (2013), basically detects combined temporal jumps of the magnetic field intensity, proton density, and bulk speed. It provides a quality factor proportional to a weighted average of the above jumps. A more technical description of this detection algorithm is given by Maksimovic et al. (2015).

The RPW shock detection algorithm has been activated in space and has provided 388 events between October 5, 2020 and April 16, 2021. It is evident that not all of these events are interplanetary shocks, but more likely discontinuities in the solar wind that need to be analyzed in greater detail. There are two main reasons for which these events are most probably not interplanetary shocks. Firstly, the SWA instrument has not operated at all (or only rarely) during this period of time. Secondly, the algorithm detection parameters (e.g., the temporal duration of the detection window) have not been set properly for this early phase of the mission. Nevertheless, we present a more detailed analysis of one of these events below in order to present the capabilities of the instrument.

Figure 3 shows one example of such discontinuity, detected by RPW on December 20, 2020 at 06:11:37.74 UT (origin of the 

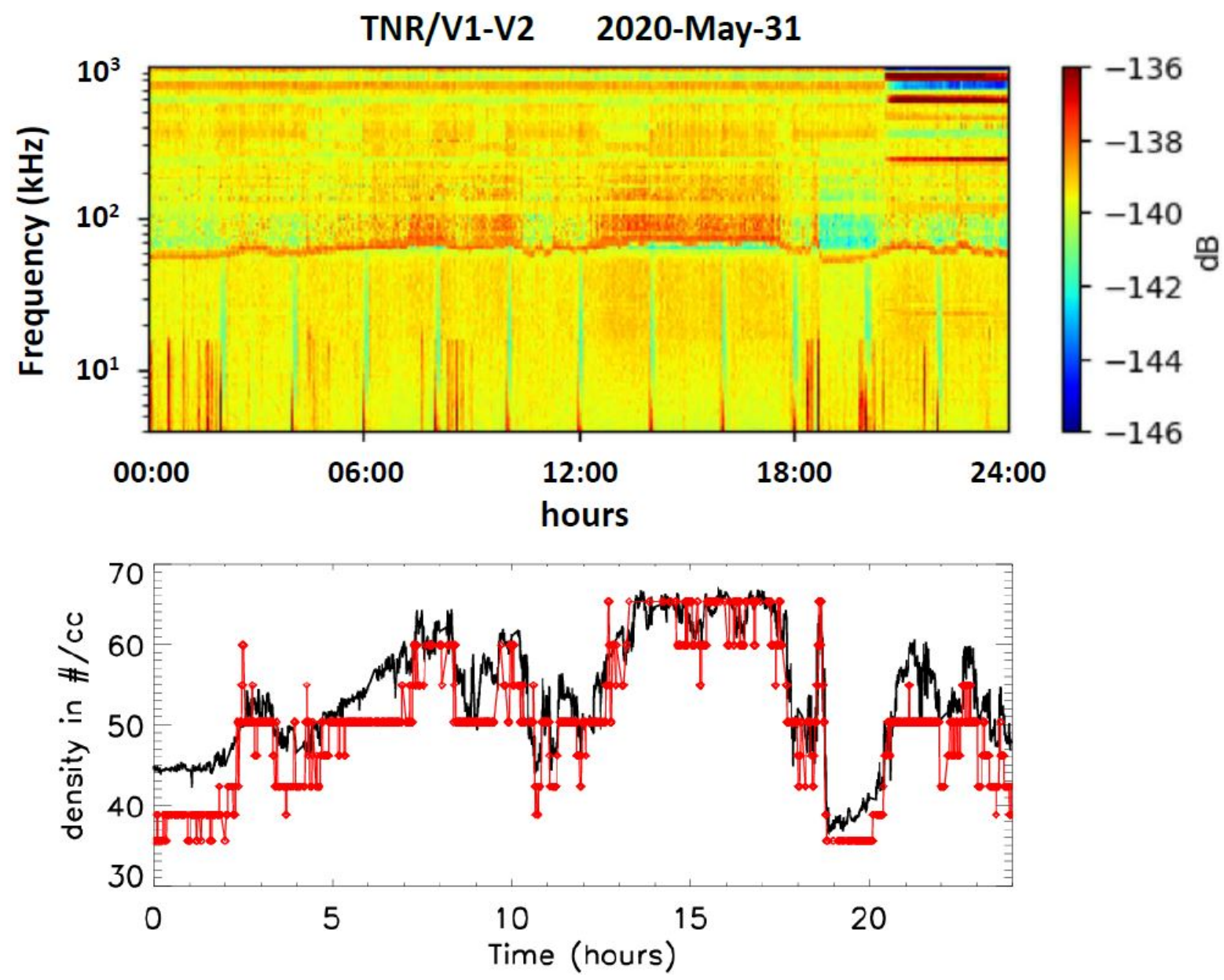

Fig. 2. Upper panel: TNR dynamic spectrum of the $V 1-V 2$ (PZ-PY) dipole signal. The plasma frequency can clearly be seen and varies between roughly 55 and $75 \mathrm{kHz}$. Using a peak tracking technique it is possible to detect the plasma frequency and deduce the temporal variation of the local electron density $N_{\mathrm{e}-f_{\mathrm{p}}}$ displayed in red on the lower panel. Superimposed in black on the lower panel is the electron density $N_{\mathrm{e}-\mathrm{S} / \mathrm{C}}$ deduced from the spacecraft potential (Khotyaintsev et al. 2021), after calibration to $N_{\mathrm{e}-f_{\mathrm{p}}}$ over longer time interval.

time in the figure). In panel a, we display the magnetic field, $B$, data of MAG instrument in the burst mode (blue line) and the electron density $N_{\mathrm{e}}=N_{\mathrm{e}-\mathrm{S} / \mathrm{C}}$ (black line) determined from the spacecraft potential (Khotyaintsev et al. 2021). A clear anticorrelation between the norm of the field $B$ and $N_{\mathrm{e}}$ is observed. The panel $b$ shows the SCM magnetic field fluctuations within the frequency range $[5,10] \mathrm{Hz}$ in the RTN frame. At these frequencies, a wave emission is observed at around $-3 \mathrm{~s}$, just before the discontinuity front, where $B$ shows its minimum and $N_{\mathrm{e}}$ has its maximum (see see the two vertical dotted lines). To characterize this wave emission, we display a zoom of a few seconds just before the discontinuity. The panels $\mathrm{c}$ and $\mathrm{d}$ on Fig. 3 show normalized density $\delta N_{\mathrm{e}} / N_{\mathrm{e} 0}$ and magnetic field $\delta \boldsymbol{B} / B_{0}$ fluctuations within the $[5,10] \mathrm{Hz}$ frequency range, respectively. $\delta \boldsymbol{B}(t)$ is projected in the local minimum variance frame determined for the time interval between the two vertical dotted lines $[-3.8,-3] \mathrm{s}$, where the localized wave packet is observed. The normalized density is one order of magnitude smaller than the normalized field fluctuations, $\delta N_{\mathrm{e}} / N_{\mathrm{e} 0} \ll \delta B / B_{0}$, that is, the wave packet is nearly incompressible. The panel e gives a hodogram of $\delta B_{\text {med }}$ versus $\delta B_{\max }$ for this $0.8 \mathrm{~s}$ interval. The diamond indicates the start time.

The angle between the minimal variance direction, which is a proxy for the wave-vector, $\boldsymbol{k}$, and the local mean field is $\Theta_{k B}=$ $4.2^{\circ}$. The local mean field in the minimal variance (MV) frame is $\boldsymbol{B}_{0}^{\mathrm{MV}}=[0.27,0.26,5.14] \mathrm{nT}$. The local electron cyclotron frequency $f_{\text {ce }}$ is $144 \mathrm{~Hz}$. Thus, we observe a right-handed circularly polarized wave, in quasi-parallel propagation to the field, at $[0.035,0.070] f / f_{\text {ce }}$ in the satellite frame. The observed properties are in agreement with parallel whistler waves, typical for the solar wind plasma (Lacombe et al. 2014; Tong et al. 2019a).

If, indeed, whistlers are shown to be commonly observed in the solar wind, their origin and role in the thermodynamics of the solar wind, and especially of the electrons, are intensely debated (Lacombe et al. 2014; Kajdič et al. 2016; Stansby et al. 2016; Roberts et al. 2017; Tong et al. 2019b; Kuzichev et al. 2019; Vasko et al. 2019, 2020; Jagarlamudi et al. 2020). In our case, we observe parallel whistlers just in the vicinity of the discontinuity, which is possibly a slow shock wave. The presence of parallel whistlers in the foot of the shock front may suggest that this shock is supercritical, namely, that its Mach number is larger than a critical Mach number at which particles can be strongly heated and accelerated.

Finally, we note that several of the examples of whistler waves detected by LFR and reported by Chust et al. (2021) are also observed within solar wind streams with the $B, N_{\mathrm{e}}$ anticorrelation and close to the discontinuities. A more detailed study of this kind of discontinuities and associated waves will be the topic of a future study. 

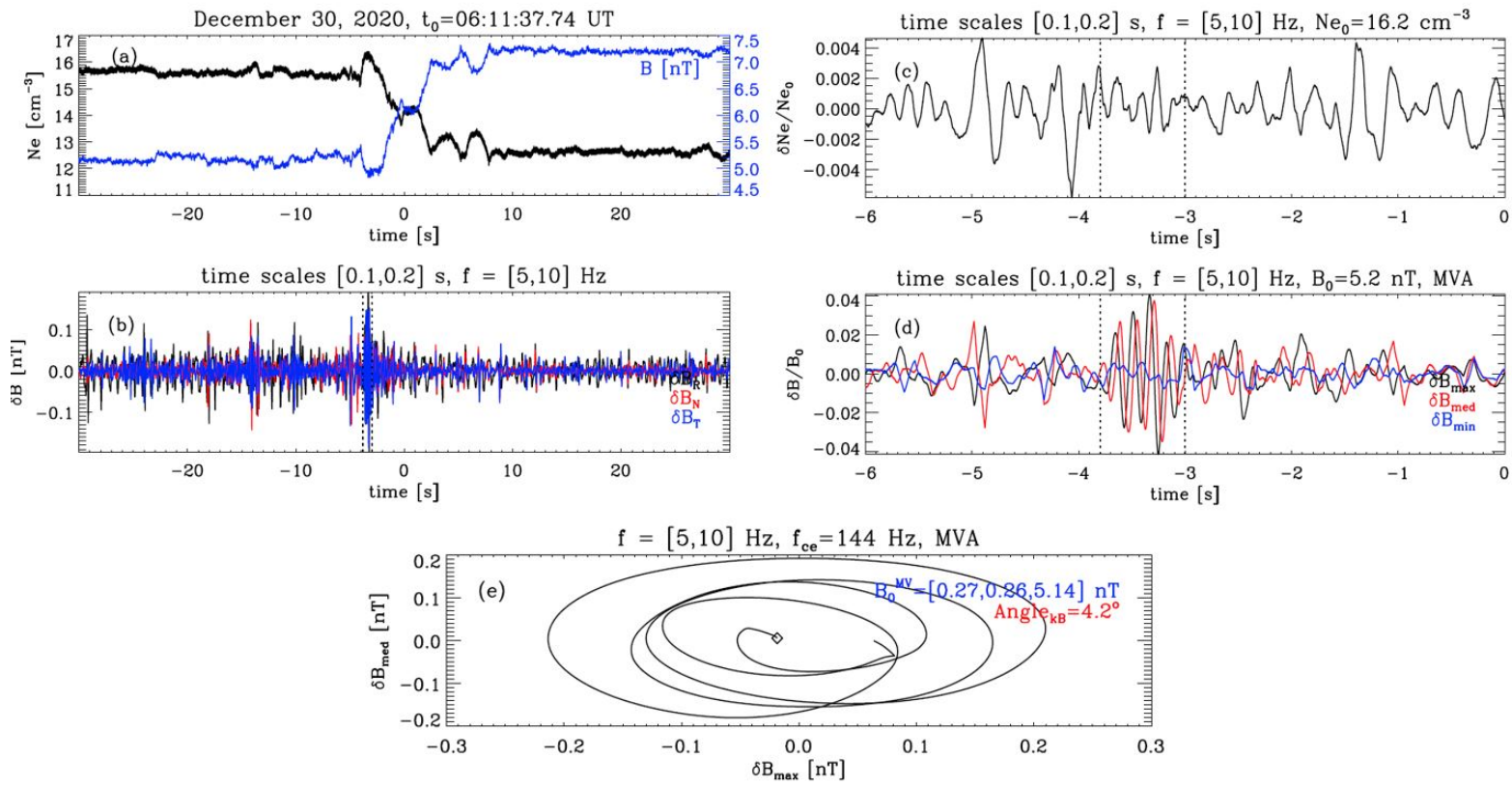

Fig. 3. Example of a discontinuity automatically detected by SBM1 mode on-board on December 30, 2020, one minute time interval around 06:11:37.74 UT. $a$ : MAG magnetic field (blue line) and electron density (in black) from RPW; $b$ : SCM magnetic fluctuations $\delta \boldsymbol{B}$ for [5, 10] Hz frequency range in the RTN frame, two vertical dotted lines indicate a localized wave emission; $c$ : density fluctuations within the same frequency range, normalized to its mean $\delta N_{\mathrm{e}} / N_{\mathrm{e} 0}$ within a $6 \mathrm{~s}$ time interval before the discontinuity; $d$ : normalized magnetic fluctuations $\delta \boldsymbol{B} / B_{0}$ in the minimum variance frame for the same time interval as $(c) ; e$ : polarization of the wave packet, diamond indicates the starting time point $-3.8 \mathrm{~s}$.

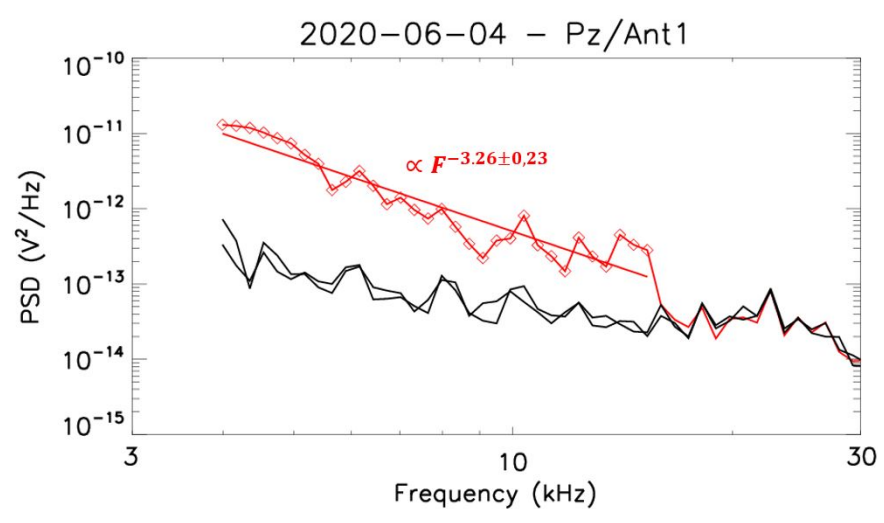

Fig. 4. Typical TNR spectrum observed during a dust impact on the spacecraft body (in red). This particular spectrum has been recorded on June 4, 2020 at 02:07:11.91. The two spectra in black, which are typical of the shot noise detected by RPW monopoles are the spectra measured at the two adjacent times, $17 \mathrm{~s}$ before and after the above mentioned time.

\subsection{Encounter with the tail of Comet ATLAS}

Despite very early in the mission timeline and occurring during commissioning of the payload, the predicted encounter with the tail of Comet C/2019 Y4 (ATLAS) at the beginning of June 2020 (Jones et al. 2020) motivated a specific campaign of the Solar Orbiter in situ instruments, exceptionally scheduled before the official start of the cruise phase. Using data from all in situ instruments, Matteini et al. (2021) have been able to identify a strong candidate for the tail crossing event: a region of very low magnetic field intensity and high plasma density, associated with a deceleration of the flow and surrounded by regions of reversed magnetic polarity interpreted as the draping structure of the comet magnetotail. The identified period of the tail crossing corresponds to a later time with respect to the initial prediction
(Jones et al. 2020), but is consistent with its revision based on the very low speed of the plasma observed by Solar Orbiter near the heliospheric current sheet and also possibly due to the fragmentation of comet ATLAS. This event is also characterised by an intense and extended wave activity at ion-scales, with various non-linear properties such as large amplitude wave-packets and static unidimensional pressure-balanced structures (e.g., mirror modes), which are interpreted as having been generated by local instabilities of cometary pick-up ions (e.g., $\mathrm{O}^{+}$).

RPW measurements of the high-cadence plasma (electron) density from the s/c potential (Khotyaintsev et al. 2021) and of the estimated flow speed, $V$, from the RPW electric field (Steinvall et al. 2021) have greatly contributed to this discovery, given that due to the time shift with respect to the expected encounter period, the detection occurred when the ion plasma instrument SWA/PAS was not operating. The RPW observations have then provided vital support for the determination of both the large-scale configuration of the comet tail (flow deceleration around the draped field, pile-up, and overall pressure balance) and the characterization of the small ion-scale waves related to pick-up ions ( $B-n$ correlation, compressibility).

\section{First measurements of the interplanetary dust flux by the TNR}

Spaceborne radio instruments are usually sensitive to dust impacts via the corresponding plasma cloud and pickup signal on the electric field antennae. This technique has been used to measure dust impacts by similar instrumentation on the Voyager probe at Saturn (Gurnett et al. 1983) and Uranus (Meyer-Vernet et al. 1986) or, more recently, by the STEREO/WAVES instrument in the solar wind (Meyer-Vernet et al. 2009) or the Cassini spacecraft during its cruise phase (Meyer-Vernet et al. 2016) and at Saturn (Ye et al. 2018). 


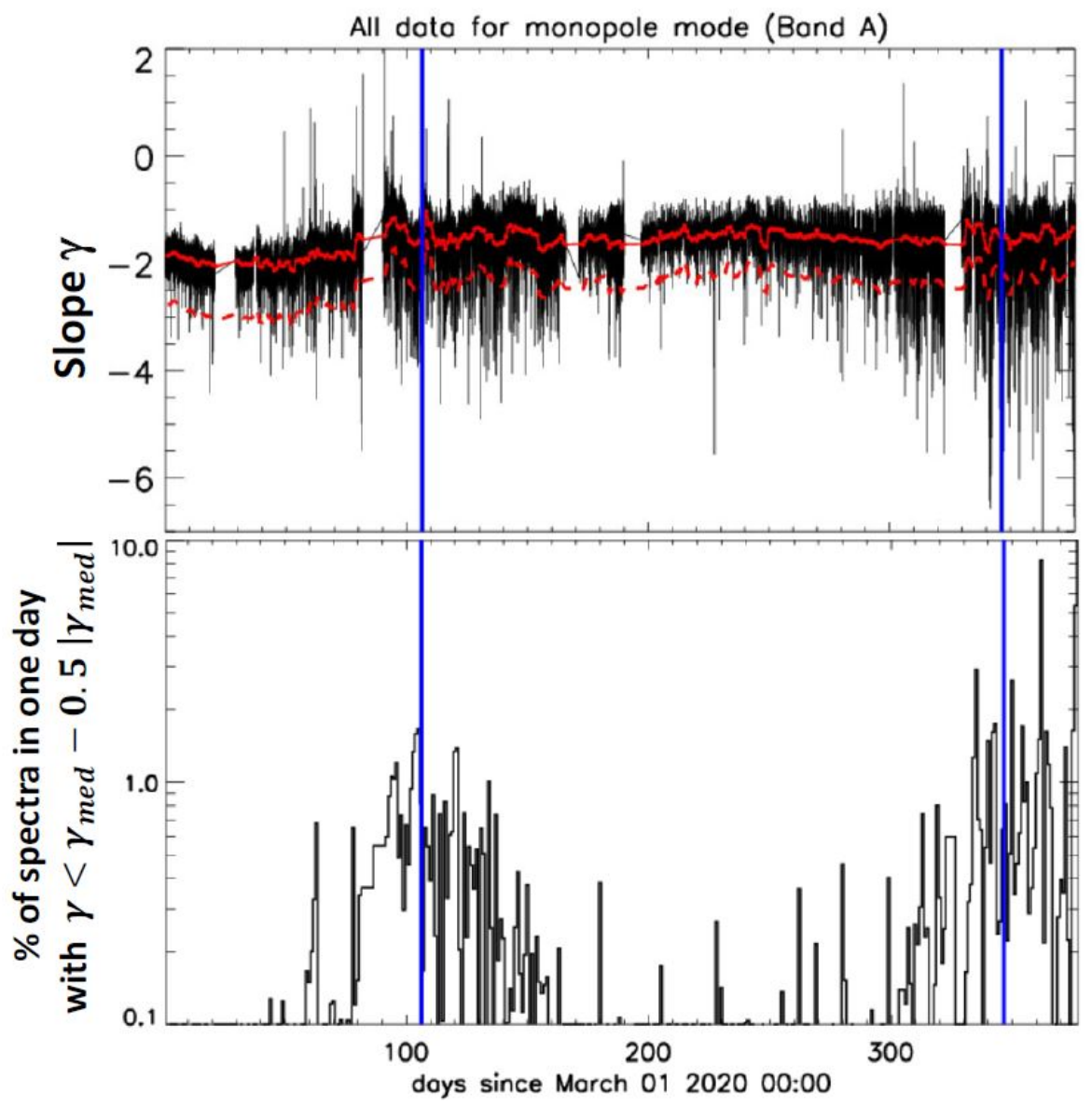

Fig. 5. Outcome of the statistical analysis of all the TNR Band A data from March 1st, 2020 to March 12th, 2021. Upper panel: power index $\gamma$ resulting from a power law fitting of the form $\mathrm{PSD}_{A}=\mathrm{PSD}_{0} \times f^{\gamma}$. The daily median values $\gamma_{\text {med }}$ of the power index $\gamma$ are displayed with the red full line. The red dashed line represents $\gamma_{\text {med }}-0.5 \times\left|\gamma_{\text {med }}\right|$. Lower panel: daily percentage of spectra with $\gamma<\gamma_{\text {med }}-0.5 \times\left|\gamma_{\text {med }}\right|$. This daily percentage is assumed to be a measure of the dust impact rate on Solar Orbiter. The vertical blue lines in both panels represent the first two perihelia of Solar Orbiter. The first one correspond to June 15, 2020 when the probe was at 0.51 au from the Sun and the second to February 10,2021 when it was at $0.49 \mathrm{au}$.
What is measured is the voltage induced on the electric antennae when a dust grain impacting the spacecraft at high velocity is vaporized and ionized, producing a plasma cloud that is partially recollected by the target. For such measurements, the effective detection area, typically the whole spacecraft surface, is much greater than the one of a classical dust particles detector.

Using data gathered by the TDS since the launch, Zaslavsky et al. (2021) have studied the dust impact rate along the Solar Orbiter's orbit. They show that the dust population studied presents a radial velocity component directed outward from the Sun, the order of magnitude of which can be estimated to be roughly $50 \mathrm{~km} \mathrm{~s}^{-1}$.

In this new study, which is complementary to the one made by Zaslavsky et al. (2021), we performed a statistical analysis of dust impacts seen by the TNR radio receiver. Indeed, when a dust particle produces a typical waveform signal as the one displayed on Fig. 1 in Zaslavsky et al. (2021), complementary measurements can be made by TNR. If the dust impact occurs during the integration time of the TNR Band A (4 to $15.3 \mathrm{kHz}$ ), the observed spectrum due to the impact is steeper than the typical $f^{-2}$ due to the electron shot noise at these frequencies (Meyer-Vernet et al. 2009, 2016). This is illustrated by Fig. 4, where a typical TNR spectrum observed during a dust impact on the spacecraft body is shown in red. This particular spectrum has been recorded on June 4, 2020 at 02:07:11.91. The two spectra in black, which are typical of the shot noise acquired by RPW monopoles are measured $17 \mathrm{~s}$ before and after the time indicated above. The dust spectrum can be fitted by the power law as $f^{-3.26 \pm 0.23}$, which is much steeper than the previous and consecutive spectra.
We have analyzed all the TNR Band A data from March 1st, 2020 to March 12th, 2021, as we did for the above example shown in Fig. 4. The upper panel of Fig. 5 displays the power index $\gamma$ resulting from a power law fitting of the form $\mathrm{PSD}_{A}=\mathrm{PSD}_{0} \times f^{\gamma}$. There are 2598489 data points in this figure covering 340 days of observations. This corresponds to about five spectra recorded each minute in monopole mode for the TNR Band A. We note that the effective integration time for a single TNR A spectrum is varying between 0.32 and $2.56 \mathrm{~s}$ for the considered period. On average, most of the spectra vary as $f^{-2}$, typical for the electron shot noise. There are however many spectra with a power law well below $\gamma=-2$. In order to quantify the amount of these spectra we have computed the daily median values $\gamma_{\text {med }}$ of the power index $\gamma$. The daily $\gamma_{\text {med }}$ values are displayed with the red full line in the upper panel of Fig. 5. The red dashed line represents $\gamma_{\text {med }}-0.5 \times\left|\gamma_{\text {med }}\right|$. Then we have computed the daily percentage of spectra with $\gamma<\gamma_{\text {med }}-0.5 \times\left|\gamma_{\text {med }}\right|$. This daily percentage, which is displayed in the lower panel of the figure is assumed to be a measure of the dust impact rate on Solar Orbiter. The vertical blue lines in Fig. 5 represent the first two perihelia of Solar Orbiter. The first one correspond to June 15 2020, when the probe was at 0.51 au from the Sun, and the second to February 10 2021, when it was at $0.49 \mathrm{au}$. When plotted as a function of the radial distance of the probe, as done in Fig. 6 one can see that this impact rate is clearly decreasing with decreasing distance to the Sun, a general behavior that is in agreement with remote and in situ measurements from Helios (Leinert et al. 1981) and Parker Solar Probe (Szalay et al. 2020; Page et al. 2020), as well as with the more detailed analysis by Zaslavsky et al. (2021) with RPW TDS on Solar Orbiter. 

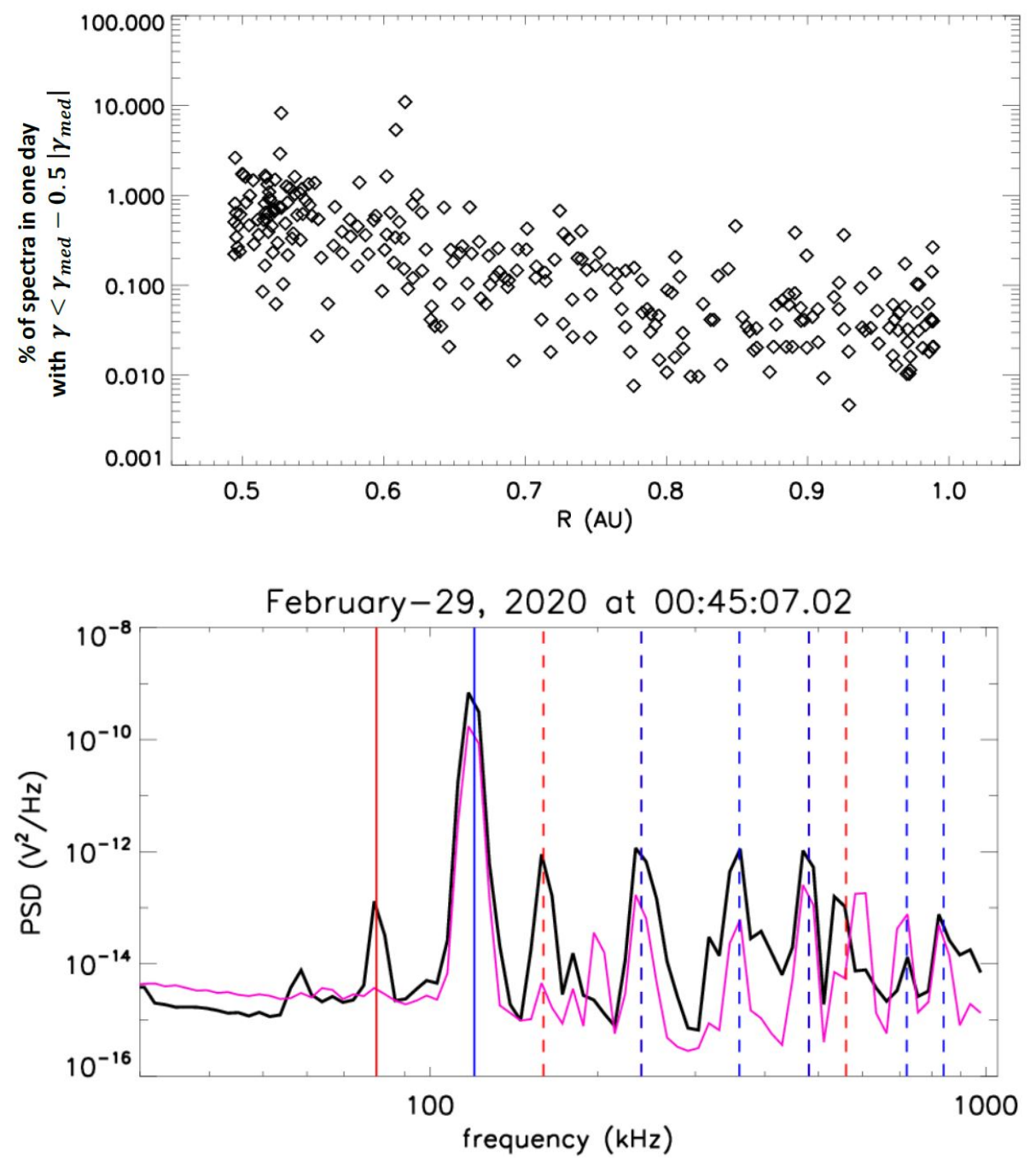

Fig. 6. Daily percentage of spectra with $\gamma<$ $\gamma_{\text {med }}-0.5 \times\left|\gamma_{\text {med }}\right|$, assumed to be a measure of the dust impact rate on Solar Orbiter, as a function of radial distance.
Fig. 7. Typical TNR spectrum in monopole mode, recorded on February 29, 2020 at 00:45:07.02. The PCDU and RW spurious peaks are indicated by the blue and red vertical lines, respectively. In addition, we display some of the harmonics $(2,3,46$, and 7 for the $120 \mathrm{kHz}$ and $2,3,6$, and 7 for the $80 \mathrm{kHz}$ ) of these two frequencies with dashed vertical lines.

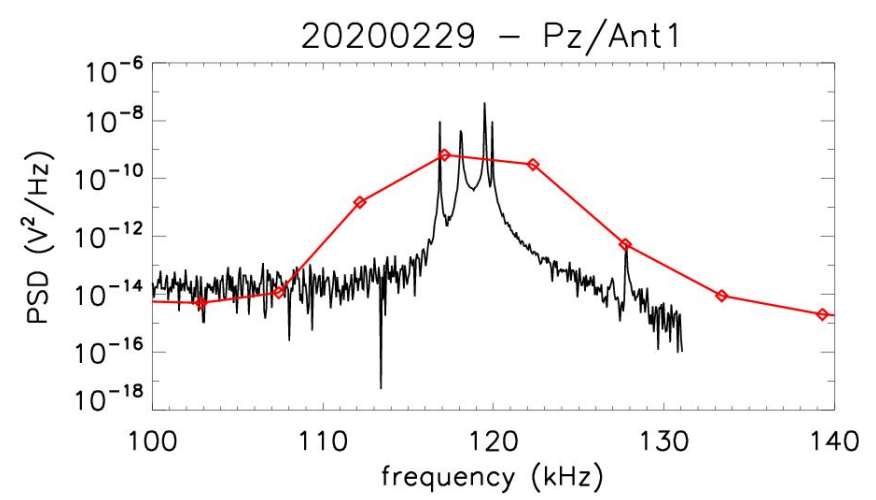

Fig. 8. Zoom around $120 \mathrm{kHz}$ of the TNR power spectrum (in red) as presented in Fig. 7. The black line, deduced from TDS waveform measurements during that time, shows four narrower peaks that correspond to the drive frequencies of the PCDU.

\section{Electro-magnetic environment of the instrument}

In this section, we describe the EM perturbations, caused mostly by the spacecraft platform and solar panels, suffered by RPW in space and which impacts its science performance. We first illustrate the most intense perturbations and then provide a summary of the contaminated frequencies, with the origin of the con- tamination and the associated science impacts. A comprehensive report on this topic was given by Maksimovic (2020).

\subsection{Electro-magnetic contamination by the spacecraft}

The most prominent perturbation, suffered by the RPW instrument, comes from the spacecraft power converter and distribution Unit (PCDU), and is radiated by the solar panels at about $120 \mathrm{kHz}$. This is due to the absence of an appropriate filtering system between the PCDU and the panels. The second permanent perturbation from the spacecraft occurs at $80 \mathrm{kHz}$ and is produced by the reaction wheels (RW) electronic box, located in the spacecraft body.

The black full line on Fig. 7 corresponds to a typical spectrum of the PZ/Ant1 monopole power spectral density as measured by the RPW Thermal Noise Receiver (TNR). The magenta full line represents the dipole spectrum (PZ-PY) recorded $17 \mathrm{~s}$ earlier. The conspicuous $120 \mathrm{kHz}$ spurious emission (blue vertical full line) can be clearly seen and is always present in the data since the antenna deployment phase and is always about $50 \mathrm{~dB}$ above the background level. Because of the persistent presence of this contamination, the internal RPW algorithm does not allow the automatic on-board detection of the plasma frequency, as was expected. Consequently, the RPW plasma frequency low-latency data, made available by ESA in the Solar Orbiter Archive ${ }^{1}$,

\footnotetext{
1 http://soar.esac.esa.int
} 


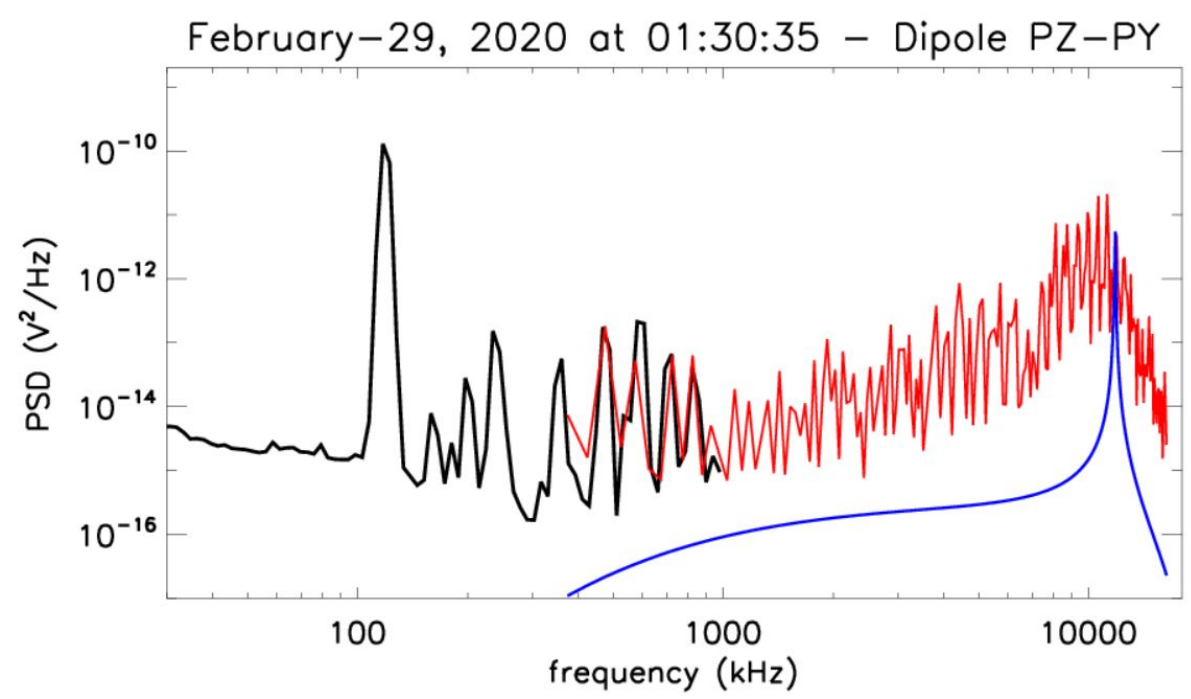

Fig. 9. Typical TNR dipole (PZ-PY) spectrum (black line) and the corresponding dipole spectrum in the HFR frequency range (red line). The HFR spectrum is very variable with frequency. A model of the galactic radio background, as it should be seen by the RPW dipole with the antenna gain factor modeled by Vecchio et al. (2021), is indicated by the blue curve. Given the frequency resolution of the HFR spectrum (192 among the 321 possible frequencies), no measurements are reaching the galactic background for this particular spectrum. cannot be used at present. A patch of the RPW flight software is planned in order to possibly correct this problem. The other spurious line that can be seen in Fig. 7 is the RW signal at $80 \mathrm{kHz}$ (red vertical full line). The amplitudes of the 80 and $120 \mathrm{kHz}$ peaks are always larger when seen by TNR in monopole mode than in the dipole one. It is, in fact, the $80 \mathrm{kHz}$, which probably corresponds to noise caused by the spacecraft body, is almost not visible in the dipole case.

In addition to the 80 and $120 \mathrm{kHz}$ lines, we display, in Fig. 7, some of their harmonics, illustrating how these harmonics are polluting permanently most of the TNR frequencies above $100 \mathrm{kHz}$ in both dipole and monopole modes. This contamination has a significant impact on the TNR science performance.

Looking more closely at the contamination at $120 \mathrm{kHz}$, it is possible to assume that what is observed as a single peak by the TNR is in fact made up of four to six narrower peaks that correspond to the drive frequencies of the PCDU. Figure 8 displays a zoom of the TNR spectrum (in red) around $120 \mathrm{kHz}$ on which we have superimposed the spectrum observed by the TDS instrument. The TDS spectrum is obtained from a waveform of 4096 points measuring the signal of PZ antenna, sampled at $262.1 \mathrm{kHz}$. We can clearly see that the contamination of the PCDU breaks down into four distinct peaks, that are visible thanks to the high-frequency resolution of the TDS.

At higher frequencies, in the frequency range of the high frequency receiver (HFR), the harmonics of the RW and PCDU create a background of spurious lines that unfortunately pollute the whole spectrum, as can be seen on Fig. 9. In this figure, we display a typical TNR dipole (PZ-PY) spectrum (black line) and the corresponding dipole spectrum in the HFR frequency range (red line). The HFR spectrum is quite variable with frequency. A model of the galactic radio background, as it should be seen by the RPW dipole with the antenna gain factor modeled by Vecchio et al. (2021), is indicated by the blue curve. Given the frequency resolution of the HFR spectrum (192 among the 321 possible frequencies), no measurements reach the galactic background for this particular spectrum.

In an attempt to identify the less contaminated TNR-HFR frequencies, we analyzed all measurements between March 2020 and March 2021. Figure 10a shows a histogram of the "clean" frequencies for the TNR operating in the $V 1-V 2$ dipole mode connected to channel 1 . The red and blue vertical lines are $80 \mathrm{kHz}$ and $120 \mathrm{kHz}$ harmonics, respectively. We used an automated identification algorithm which functions as follows: we first computed the daily $1 \%$ background levels for frequency channels above $100 \mathrm{kHz}$. This was done for each of the available frequency channels and for all the available data. Then we computed the Butterworth pass-band (Butterworth 1930) filtered values of all these $1 \%$ levels. We used a classical Butterworth filter of order one and a cutoff frequency of 9 . Finally, we compared the relative variations of each of the individual $1 \%$ levels with respect to the filtered values. If one individual $1 \%$ level is lower by $4 \mathrm{~dB}$ than its corresponding filtered value, then we consider this frequency channel to be "clean". The value of $4 \mathrm{~dB}$ has been chosen empirically in order to retrieve a sufficient number o "clean" frequencies for science at the end. The blue histogram in Fig. 10a shows the percentage of clean channels for one year of measurements (100\% correspond to 1626722 radio spectra). For this antenna configuration, we identified 13 clean frequencies when the percentage exceeds $50 \%$ (denoted by a black dashed line). We performed the same analysis for all TNR antenna monopole and dipole configurations (not shown here). We found only 7 out of 52 frequency channels above $100 \mathrm{kHz}$ (i.e., 13\%), which fulfill the above-mentioned criteria for all antenna-channel configurations (listed in Fig. 10a and denoted by green crosses). Figure $10 \mathrm{~b}$ displays the same analysis applied to the HFR data from the same time interval. In this case, we identified only 23 out of 321 frequency channels that are not affected by $80 / 120 \mathrm{kHz}$ EMC (i.e. 7\%). This frequency list could be used in the future, when operating the TNR-HFR, in order to skip the contaminated frequencies and to significantly improve the temporal resolution the HFR and possibly of the TNR.

We should note that for intense natural radio signals, typically above $10^{-12} V^{2} \mathrm{~Hz}$ when observed by the HFR, most of the EM contamination should be negligible for frequencies up to about $3 \mathrm{MHz}$. Given, however, that the typical distribution of Type III radio fluxes (see Fig. 3 in Maksimovic et al. 2020), this will concern only a few percent of all the events during the whole duration of the Solar Orbiter nominal phase of the mission.

In addition to the two prominent contamination reported above, several signatures of EM contamination are also visible in the TNR spectra below $80 \mathrm{kHz}$ and in the RPW Low Frequency Receiver (LFR) down to DC. This contamination is variable both in frequency and amplitude and is reported in Table 1. Their root causes are still under investigation. All the information and updates about the RPW EM contamination can be found online ${ }^{2}$.

2 https://rpw. lesia.obspm.fr/Engineering 

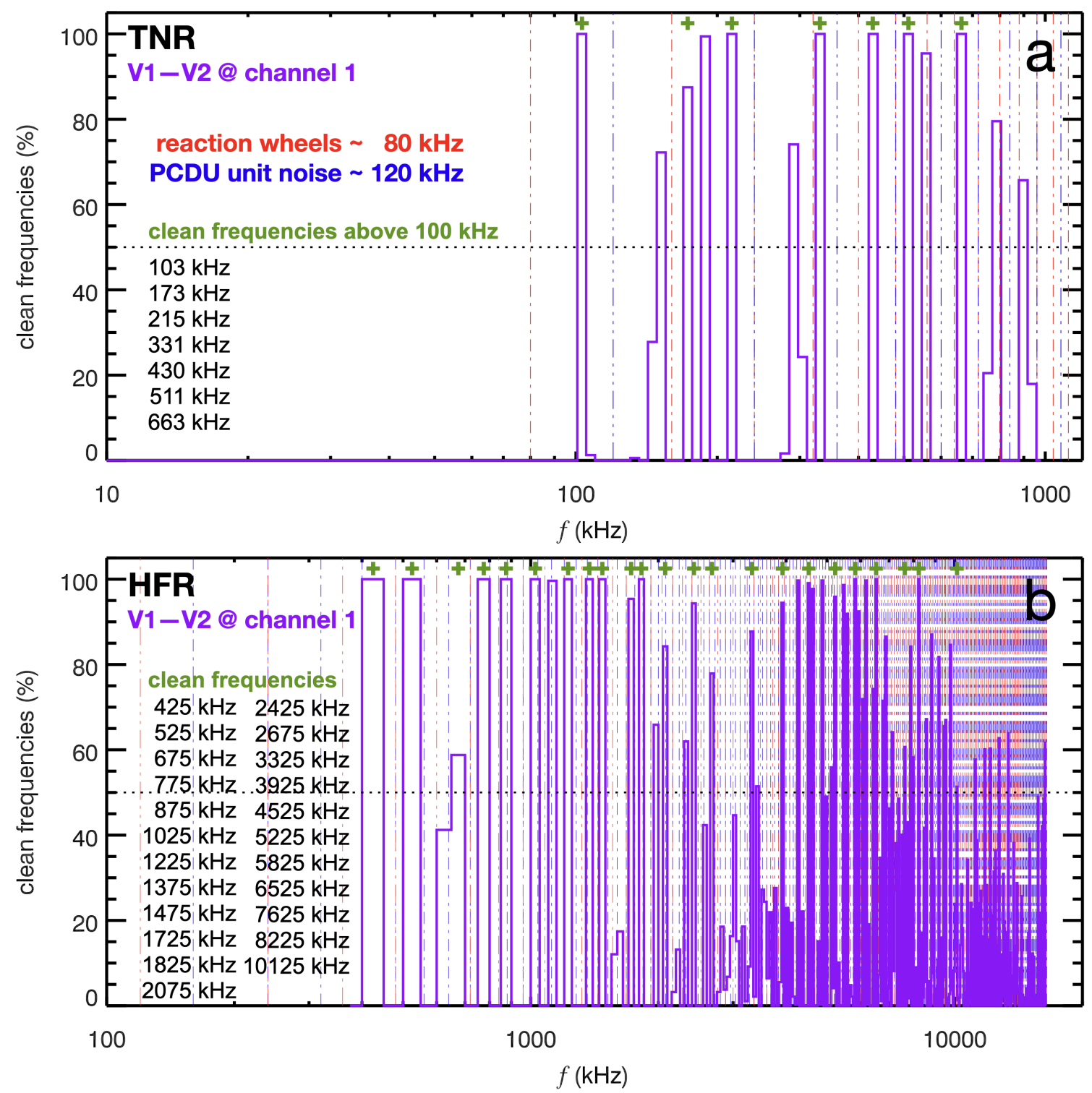

Fig. 10. Histograms of the percentages of clean channels for one year of measurements for TNR (panel a) and HFR (panel $b$ ). See text for more detail.

\subsection{RPW internal EM perturbations}

For the RPW magnetic measurements, the observations can be also contaminated by more or less regular spikes, occurring about every $90 \mathrm{~s}$ and depending on the need to heat SCM heating requirements. They are caused by the SCM heater switch on or off. The contamination duration is typically from 0.1 to $0.2 \mathrm{~s}$, with, however, all frequencies being corrupted. Figure 11 displays a zoom on the SCM heater signature for the LF1Y SCM sensor. Each color corresponds to one observation. This SCM heater contamination is not corrected in the Level 2 RPW data that are publicly available. The SCM heater switch on or off is, however, indicated in the quality flag of the relevant data. In the future, this contamination may be corrected for Level 3 data.

As of January 2021, new perturbations have appeared and concern mostly the TDS and the TNR sub-systems. These perturbations, which are still being currently assessed, appear as narrow-banded and very intense emissions at frequencies varying between 30 to about $60 \mathrm{kHz}$. This electric contamination is so intense that the TNR cannot be used to detect properly the electron plasma frequency and the internal TDS software can- not work properly to analyse the observed waves. At the time of writing this article, it is believed that these perturbations could be due to some complex interactions between the spacecraft plasma sheath and the solar wind environment. These investigations are ongoing.

\subsection{Summary of the RPW EM contamination and caveats for the users of RPW data}

Table 1 summarizes the most important electro-magnetic perturbations impacting RPW and characterized so far. Each of them has an impact on the science return of RPW, which is not easy to assess from a global point of view. For the time being, we have only assigned three levels of severity for the impact on science: low, medium, and high.

As we detail in the section above, most of the perturbations summarized in Table 1 are caused by the spacecraft platform. Except for the perturbation labeled 12, none of them can be mitigated in flight. Therefore, the mitigation process, when possible, will have to be implemented by the RPW team either by 
Table 1. Summary of the most important electro-magnetic perturbations impacting RPW and characterized so far.

\begin{tabular}{|c|c|c|c|c|c|c|}
\hline Label & Description & $\begin{array}{l}\text { Corrupted } \\
\text { frequencies }\end{array}$ & $\begin{array}{l}\text { Sensor } \\
E / B\end{array}$ & $\begin{array}{l}\text { Root } \\
\text { cause }\end{array}$ & $\begin{array}{l}\text { Impacted } \\
\text { sub-systems }\end{array}$ & $\begin{array}{l}\text { Severity and } \\
\text { mitigations }\end{array}$ \\
\hline 1 & Continuous emission & $\sim 1.3 \mathrm{~Hz}$ & $B$ & Unknown & SCM, LFR & TBD \\
\hline 2 & $\begin{array}{l}\text { Pulse of the Attitude } \\
\text { and Orbit Control Sys- } \\
\text { tem (AOCS) synchro- } \\
\text { nization }\end{array}$ & 8 and $16 \mathrm{~Hz}$ & $B$ & S/C AOCS & LFR & Low severity \\
\hline 3 & SCM heater & $\leq 100 \mathrm{~Hz}$ & $B$ & SCM & LFR & $\begin{array}{l}\text { Low severity, flagging the } \\
\text { data }\end{array}$ \\
\hline 4 & $36 \mathrm{~Hz}$ and harmonics & $\leq 200 \mathrm{~Hz}$ & $E$ & Unknown & LFR & Medium severity \\
\hline 5 & $\begin{array}{l}3 \mathrm{~h} \text { periodicity pertur- } \\
\text { bation in LF }\end{array}$ & $\leq 500 \mathrm{~Hz}$ & $B$ & Unknown & LFR & TBD \\
\hline 6 & EUI science mode & $\begin{array}{l}\text { Broadband up to } \\
1.5 \mathrm{kHz}\end{array}$ & $E$ and $B$ & EUI & LFR & $\begin{array}{l}\text { Medium severity, should } \\
\text { be taken care of during } \\
\text { operations }\end{array}$ \\
\hline 7 & Solo-HI $6.3 \mathrm{kHz}$ & $\sim 6.3 \mathrm{kHz}$ & $E$ and $B$ & Solo-HI & LFR & $\begin{array}{l}\text { Medium severity, should } \\
\text { be taken care of during } \\
\text { operations }\end{array}$ \\
\hline 8 & $\sim 50 \mathrm{kHz}$ & $\begin{array}{l}\text { From } \sim 30 \text { to } \\
\sim 60 \mathrm{kHz}\end{array}$ & $E$ & Unknown & $\begin{array}{l}\text { TNR-HFR and } \\
\text { TDS }\end{array}$ & High severity \\
\hline 9 & $80 \mathrm{kHz}$ & $\begin{array}{l}\sim 80 \mathrm{kHz} \text { and } \\
\text { harmonics }\end{array}$ & $E$ & $\begin{array}{l}\mathrm{S} / \mathrm{C} \text { reaction wheels } \\
\text { electronics }\end{array}$ & TNR-HFR & $\begin{array}{l}\text { High severity, mitigation } \\
\text { by RPW team }\end{array}$ \\
\hline 10 & $120 \mathrm{kHz}$ & $\begin{array}{l}\sim 120 \mathrm{kHz} \text { and } \\
\text { harmonics }\end{array}$ & $E$ and $B$ & $\begin{array}{l}\text { S/C PCDU noise radi- } \\
\text { ated by SA }\end{array}$ & $\begin{array}{l}\text { TNR-HFR and } \\
\text { TDS }\end{array}$ & $\begin{array}{l}\text { High severity, mitigation } \\
\text { by RPW team }\end{array}$ \\
\hline 11 & $\sim 250 \mathrm{kHz}$ & $244.6,255.5 \mathrm{kHz}$ & $B$ & MAG heater & TNR & $\begin{array}{l}\text { Low severity, mitigation } \\
\text { by flagging the data }\end{array}$ \\
\hline 12 & $\sim 300 \mathrm{kHz}$ & $\begin{array}{l}\text { Several } \\
\text { frequencies } \\
\text { around } 300 \mathrm{kHz}\end{array}$ & $E$ & $\mathrm{~S} / \mathrm{C}$ battery charging & TNR & $\begin{array}{l}\text { High severity if not mit- } \\
\text { igated by ESA during } \\
\text { operations }\end{array}$ \\
\hline
\end{tabular}

Notes. Their root causes are still under investigation. All the information and updates about the RPW EM contamination can be found online (https://rpw. lesia.obspm.fr/Engineering).

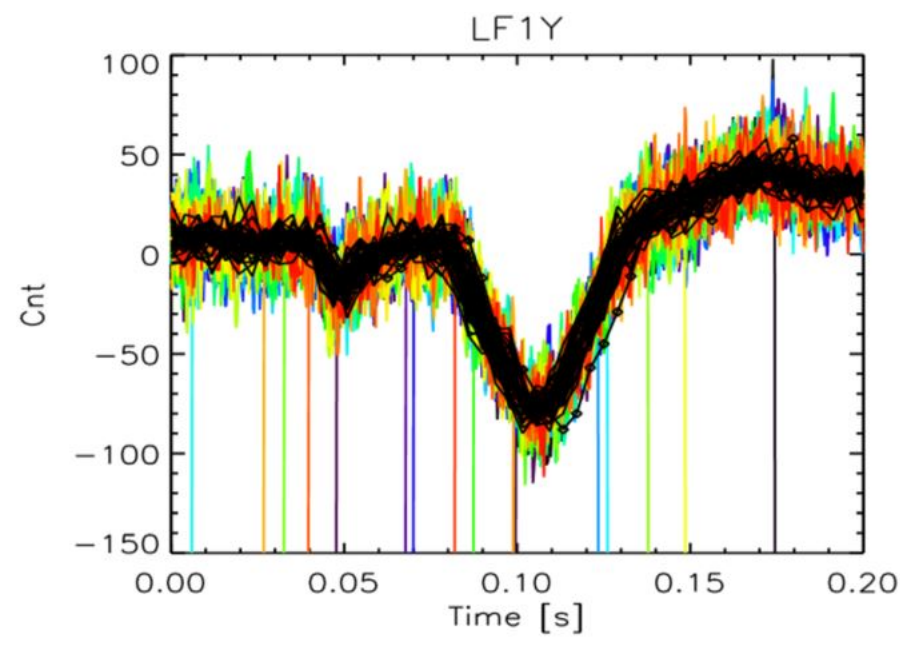

Fig. 11. Zoom on the SCM heater signature on the LF1Y antenna. Each color corresponds to one observation. The vertical lines are caused by the plotting procedure and are not due to SCM signal variations.

post-processing of the data or by forthcoming changes of the RPW flight software.

Regarding the EM contamination by other instruments, it is possible that it exists but actually it is masked by the contamination caused by the platform. At the moment, RPW is only contaminated by emissions caused by three instruments, namely: the Extreme Ultraviolet Imager (EUI, Rochus et al. 2020), the Solar Orbiter Heliospheric Imager (Solo-HI, Howard et al. 2020), and the magnetometer MAG (Horbury et al. 2020). These perturbations, labeled 6,7 , and 11 have low or medium severity impacts on the RPW measurements.

Finally, Table 1 constitutes a reference for future users of the RPW data archived at ESA. Any natural phenomenon observed at frequencies including those indicated on this table should be considered suspicious and should require further analysis. In this type of situation, it is strongly recommended to contact the persons responsible for the respective RPW data production (the relevant documentation can be found online ${ }^{3}$ ).

\section{Summary}

In this article, we present the first and numerous RPW observations gathered thus far in order to assess the instrument's scientific performance. Despite several electro-magnetic disturbances that impact the instrument, the data it provides are of sufficient quality to achieve most of the scientific objectives that have been assumed. At the time of writing, the entire RPW team had already launched preparations for the start of the nominal phase of the mission, which will begin next November. We are confident that RPW will fulfill its science objectives and that it will continue to provide excellent data.

Acknowledgements. Solar Orbiter is a mission of international cooperation between ESA and NASA, operated by ESA. The RPW instrument has been designed and funded by CNES, CNRS, the Paris Observatory, The Swedish National Space Agency, ESA-PRODEX and all the participating institutes.

3 http://soar.esac.esa.int 
J. Rodriguez-Pacheco wishes to thank FEDER/MCIU-AEI/Project ESP201788436-R. R. F. Wimmer-Schweingruber acknowledges support by the German Space Agency (DLR) under grant 50OT2002. V. Krupař acknowledges the support by NASA under grants 18-2HSWO218_2-0010 and 19-HSR-19_2-0143.

\section{References}

Alexandrova, O., Chen, C. H. K., Sorriso-Valvo, L., Horbury, T. S., \& Bale, S D. 2013, Space Sci. Rev., 178, 101

Belcher, J. W., \& Davis, L., Jr. 1971, J. Geophys. Res., 76, 3534

Berčič, L., Verscharen, D., Owen, C. J., et al. 2021, A\&A, 656, A31 (SO Cruise Phase SI)

Bruno, R., \& Carbone, V. 2013, Liv. Rev. Sol. Phys., 10, 2

Butterworth, S. 1930, Experimental Wireless and the Wireless Engineer, 7, 536

Carbone, F., Sorriso-Valvo, L., Khotyaintsev, Yu. V., et al. 2021, A\&A, 656, A16 (SO Cruise Phase SI)

Chust, T., Kretzschmar, M., Graham, D. B., et al. 2021, A\&A, 656, A17 (SO Cruise Phase SI)

de Hoffmann, F., \& Teller, E. 1950, Phys. Rev., 80, 692

Dimmock, A., Khotyaintsev, Yu. V., Lalti, A., et al. 2021, A\&A, submitted (SO Cruise Phase SI)

Gómez-Herrero, R., Pacheco, D., Kollhoff, A., et al. 2021, A\&A, 656, L3 (SO Cruise Phase SI)

Graham, D. B., Khotyaintsev, Yu. V., Vaivads, A., et al. 2021, A\&A, 656, A23 (SO Cruise Phase SI)

Greco, A., Matthaeus, W. H., Servidio, S., Chuychai, P., \& Dmitruk, P. 2009, ApJ, 691, L111

Gurnett, D. A., Grun, E., Gallagher, D., Kurth, W. S., \& Scarf, F. L. 1983, Icarus, 53, 236

Hadid, L. Z., Edberg, N. J. T., Chust, T., et al. 2021, A\&A, 656, A18 (SO Cruise Phase SI)

Horbury, T. S., O’Brien, H., Carrasco Blazquez, I., et al. 2020, A\&A, 642, A9

Howard, R. A., Vourlidas, A., Colaninno, R. C., et al. 2020, A\&A, 642, A13

Issautier, K., Meyer-Vernet, N., Moncuquet, M., Hoang, S., \& McComas, D. J. 1999, J. Geophys. Res.: Space Phys., 104, 6691

Jagarlamudi, V. K., Alexandrova, O., Berčič, L., et al. 2020, ApJ, 897, 118

Jones, G. H., Afghan, Q., \& Price, O. 2020, Res. Notes Am. Astron. Soc., 4 62

Kajdič, P., Alexandrova, O., Maksimovic, M., Lacombe, C., \& Fazakerley, A. N. 2016, ApJ, 833, 172

Khotyaintsev, Yu. V., Graham, D. B., Vaivads, A., et al. 2021, A\&A, 656, A19 (SO Cruise Phase SI)

Kretzschmar, M., Chust, T., Krasnoselskikh, V., et al. 2021, A\&A, 656, A24 (SO Cruise Phase SI)

Kruparova, O., Maksimovic, M., Šafránková, J., et al. 2013, J. Geophys. Res. Space Phys., 118, 4793

Kuzichev, I. V., Vasko, I. Y., Rualdo Soto-Chavez, A., et al. 2019, ApJ, 882, 81

Lacombe, C., Alexandrova, O., Matteini, L., et al. 2014, ApJ, 796, 5

Leinert, C., Richter, I., Pitz, E., \& Planck, B. 1981, A\&A, 103, 177

Lion, S., Alexandrova, O., \& Zaslavsky, A. 2016, ApJ, 824, 47

Maksimovic, M. 2020, RPW-SCI-TSR-001894-LES - EMC Impact on the RPW Science Performance in Space, https://rpw.lesia.obspm.fr/ Engineering

Maksimovic, M., Hoang, S., Meyer-Vernet, N., et al. 1995, J. Geophys. Res., 100, 19881

Maksimovic, M., Bougeret, J.-L., Perche, C., et al. 1998, Geophys. Res. Lett., 25,1265

Maksimovic, M., Kruparova, O., \& Alexandrova, O. 2015, RPW-SCI-NTT000243-LES - RPW Instrument: Definition of the In-Flight Burst Modes Detection Algorithms, https://rpw. lesia.obspm. fr/Engineering

Maksimovic, M., Bale, S. D., Chust, T., et al. 2020, A\&A, 642, A12

Maksimovic, M., Bale, S. D., Chust, T., et al. 2021, A\&A, 654, C2

Matteini, L., Laker, R., Horbury, T., et al. 2021, A\&A, 656, A39 (SO Cruise Phase SI)

Meyer-Vernet, N., Aubier, M. G., \& Pedersen, B. M. 1986, Geophys. Res. Lett., 13,617

Meyer-Vernet, N., Maksimovic, M., Czechowski, A., et al. 2009, Sol. Phys., 256, 463

Meyer-Vernet, N., Moncuquet, M., Issautier, K., \& Schippers, P. 2016, J. Geophys. Res.: Space Phys., 122, 8

Meyer-Vernet, N., Issautier, K., \& Moncuquet, M. 2017, J. Geophys. Res., 122, 7925

Müller, D., St. Cyr, O. C., Zouganelis, I., et al. 2020, A\&A, 642, A1

Musset, S., Maksimovic, M., Kontar, E., et al. 2021, A\&A, 656, A34 (SO Cruise Phase SI)

Owen, C. J., Bruno, R., Livi, S., et al. 2020, A\&A, 642, A16
Page, B., Bale, S. D., Bonnell, J. W., et al. 2020, ApJS, 246, 51

Píša, D., Souček, J., Santolík, O., et al. 2021, A\&A, 656, A14 (SO Cruise Phase $\mathrm{SI})$

Roberts, O. W., Alexandrova, O., Kajdič, P., et al. 2017, ApJ, 850, 120

Rochus, P., Auchère, F., Berghmans, D., et al. 2020, A\&A, 642, A8

Rodríguez-Pacheco, J., Wimmer-Schweingruber, R. F., Mason, G. M., et al. 2020, A\&A, 642, A7

Sonnerup, B. U. O., Papamastorakis, I., Paschmann, G., \& Luehr, H. 1987, J. Geophys. Res., 92, 12137

Soucek, J., Píša, D., Kolmasova, I., et al. 2021, A\&A, 656, A26 (SO Cruise Phase SI)

Sperveslage, K., Neubauer, F. M., Baumgärtel, K., \& Ness, N. F. 2000, Nonlinear Process. Geophys., 7, 191

Stansby, D., Horbury, T. S., Chen, C. H. K., \& Matteini, L. 2016, ApJ, 829, L16

Steinvall, K., Khotyaintsev, Yu. V., Cozzani, G., et al. 2021, A\&A, 656, A9 (SO Cruise Phase SI)

Szalay, J. R., Pokorný, P., Bale, S. D., et al. 2020, ApJS, 246, 27

Tong, Y., Vasko, I. Y., Artemyev, A. V., Bale, S. D., \& Mozer, F. S. 2019a, ApJ, 878,41

Tong, Y., Vasko, I. Y., Pulupa, M., et al. 2019b, ApJ, 870, L6

Tsurutani, B. T., Lakhina, G. S., Verkhoglyadova, O. P., et al. 2011, J. Geophys. Res.: Space Phys., 116, A02103

Tu, C. Y., \& Marsch, E. 1995, Space Sci. Rev., 73,

Vaivads, A., Eriksson, A. I., André, M., et al. 2007, Adv. Space Res., 39, 1502

Vasko, I. Y., Krasnoselskikh, V., Tong, Y., et al. 2019, ApJ, 871, L29

Vasko, I. Y., Kuzichev, I. V., Artemyev, A. V., et al. 2020, Phys. Plasmas, 27, 082902

Vecchio, A., Maksimovic, M., Krupar, V., et al. 2021, A\&A, 656, A33 (SO Cruise Phase SI)

Ye, S. Y., Kurth, W. S., Hospodarsky, G. B., et al. 2018, Geophys. Res. Lett., 45, 10,101

Zaslavsky, A., Mann, I., Soucek, J., et al. 2021, A\&A, 656, A30 (SO Cruise Phase SI)

Zouganelis, I., De Groof, A., Walsh, A. P., et al. 2020, A\&A, 642, A3

1 LESIA, Observatoire de Paris, Université PSL, CNRS, Sorbonne Université, Université de Paris, 5 Place Jules Janssen, 92195 Meudon, France

e-mail: milan.maksimovic@obspm.fr

2 Institute of Atmospheric Physics, Czech Academy of Sciences, Prague 14131, Czech Republic

3 LPP, CNRS, Ecole Polytechnique, Sorbonne Université, Observatoire de Paris, Université Paris-Saclay, Palaiseau, Paris, France

4 Swedish Institute of Space Physics (IRF), Uppsala, Sweden

5 LPC2E, CNRS, 3A Avenue de la Recherche Scientifique, Orléans, France

6 Université d'Orléans, Orléans, France

7 Space Sciences Laboratory, University of California, Berkeley, CA, USA

8 Physics Department, University of California, Berkeley, CA, USA

9 Stellar Scientific (now HELIOSPACE), 932 Parker St Suite 2, Berkeley, CA 94710, USA

10 Department of Space and Plasma Physics, School of Electrical Engineering and Computer Science, Royal Institute of Technology, Stockholm, Sweden

11 Goddard Planetary Heliophysics Institute, University of Maryland, Baltimore County, Baltimore, MD, USA

12 Heliophysics Science Division, NASA Goddard Space Flight Center, Greenbelt, MD, USA

13 CNES, 18 Avenue Edouard Belin, 31400 Toulouse, France

14 Department of Physics, Imperial College London, South Kensington Campus, London SW7 2AZ, UK

15 Technische Universität Dresden, Würzburger Str. 35, 01187 Dresden, Germany

16 Space Research Institute, Austrian Academy of Sciences, Graz, Austria

17 Faculty of Mathematics and Physics, Charles University, Prague, Czech Republic

18 Astronomical Institute, Czech Academy of Sciences, 14100 Prague, Czech Republic 
19 LAM, Pythéas, Site de Château Gombert, Marseille, France

${ }^{20}$ Nexeya Conseil et Formation, 5 Rue Boudeville ZI de Thibaud, 31100 Toulouse, France

21 Altran Sud Ouest 4, Avenue Didier Daurat, 31700 Blagnac, France

22 Université de Rennes, 1 - Avenue du Général Leclerc, 35042 Rennes, France

23 Department of Physics and Astronomy, University of Calgary, Calgary, Alberta, Canada

${ }^{24}$ School of Physics and Astronomy, University of Minnesota, Minneapolis, MN, USA

25 School of Astronomy and Astrophysics, Glasgow University, Glasgow, UK

26 Radboud Radio Lab., Department of Astrophysics/IMAPP Radboud University, PO Box 9010, 6500 GL Nijmegen, The Netherlands
27 IRAP, CNRS, Université de Toulouse, UPS, Toulouse, France

${ }^{28}$ Lunar and Planetary Laboratory, University of Arizona, Tucson, AZ 85719, USA

29 Department of Astronomy, Faculty of Mathematics, University of Belgrade, Studentski Trg 16, 11000 Belgrade, Serbia

${ }^{30}$ ESA, ESTEC, Noordwijk, The Netherlands

31 Mullard Space Science Laboratory, University College London, Holmbury St. Mary, Dorking RH5 6NT, UK

32 Space Research Group, Universidad de Alcala, Alcala de Henares, Spain

33 ESA, ESAC, Madrid, Spain

34 Institute of Experimental and Applied Physics, Christian-AlbrechtsUniversity, Kiel, Germany

35 Commission for Astronomy, Austrian Academy of Sciences, Graz, Austria 